\title{
Blood Plasma Serotonin and von Willebrand Factor as Biomarkers of Unstable Angina Progression Toward Myocardial Infarction
}

\author{
Yuliya Tyravska $^{1^{*}}$ (D), Oleksandr Savchenko ${ }^{1}$ (D), Viktor Lizogub ${ }^{1}$ (D), Nataliia Raksha ${ }^{2}$ (D), \\ Olexiy Savchuk ${ }^{2}$ iD
}

\begin{abstract}
Aim: To investigate the serotonin and von Willebrand factor (vWF) concentrations among unstable angina (UA) patients without and with progression toward myocardial infarction (outcome) and to assess the utility of both as prognostic markers of UA complications.

Materials and methods: In observational cohort study, we recruited 103 patients with ischemic heart disease (the median age 65.0 (59.0-69.0) years, 45 females (43.7\%)). After full set of investigations including high sensitive Troponin I test and 28-day follow-up period, we defined three groups: Group 1 - stable angina patients $(n=22)$ as control, Group 2 - UA patients without outcome $(n=71)$, Group 3 - UA patients with outcome $(n=10)$. We analyzed the blood plasma serotonin content by the ion-exchange chromatography with measurement of serotonin on fluorescence spectrophotometer. VWF concentration was determined by ELISA. We compared the concentrations of observed parameters among the groups with the Kruskal-Wallis test (with post-hoc Mann-Whitney test with Bonferroni-Holm correction). We assessed binary logistic models, receiver operating characteristic curves, calculated sensitivity (Se), specificity (Sp), and positive likelihood ratio (LR+) for each indicator.

Results: We registered elevation in serotonin concentration and decline in vWF concentration in Group 3 in comparison with Group 2 (22.670 (20.687-24.927) $\mu \mathrm{g} / \mathrm{ml}$ vs $11.980(8.120-15.000) \mu \mathrm{g} / \mathrm{ml}, \mathrm{p}<0.001$, and $0.117(0.109-0.120)$ rel.units $/ \mathrm{ml}$ vs $0.134(0.127-0.143)$ rel.units $/ \mathrm{ml}, \mathrm{p}<0.001)$ and Group 1 (12.340 (10.05213.619) $\mu \mathrm{g} / \mathrm{ml}, \mathrm{p}<0.001$, and 0.137 (0.127-0.156) rel.units $/ \mathrm{ml}, \mathrm{p}<0.001)$, respectively. No significant differences in serotonin and vWF concentrations between Group 1 and Group 2 were detected $(p=0.81$ and $p=0.36$, respectively). The probability of outcome increased significantly (by $60.7 \%$ and $59.7 \%$, LR+ $19.0[6.0,60.0]$ and $18.0[3.9,80.0])$ if serotonin concentration was above $21.575 \mu \mathrm{g} / \mathrm{ml}(\mathrm{Se}=80.0 \%, \mathrm{Sp}=95.8 \%, \mathrm{AUC}=0.975)$ and vWF concentration was below 0.114 rel.units $/ \mathrm{ml}(\mathrm{Se}=50.0 \%, \mathrm{Sp}=97.2 \%, \mathrm{AUC}=0.973)$, respectively.

Conclusions: Serotonin and vWF as biomarkers are demonstrated promising results for rule-in the patients with risk of short-term UA progression toward myocardial infarction.

\section{Keywords}

Unstable Angina; Myocardial Infarction; Serotonin; Factor von Willebrand

${ }^{1}$ Department of Internal Medicine \#4, Bogomolets National Medical University, Kyiv, Ukraine

${ }^{2}$ Biochemistry Department, Educational and Scientific Centre "Institute of Biology and Medicine", Taras Shevchenko National University of Kyiv, Kyiv, Ukraine

*Corresponding author: yuliya_tyravska@ukr.net
\end{abstract}

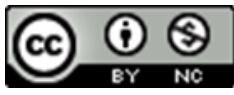




\section{Problem statement and analysis of the latest research}

Ischemic heart disease (IHD) occupies the primary position among all causes of death worldwide [1] despite the descendent trend in age-standardized acute myocardial infarction (MI) incidence and unstable angina (UA) prevalence over the past two decades [2]. Moreover, due to the overall growth of the population and its aging, the global burden of IHD has increased by $29 \%$ to 29 million disability-adjusted life years [2].

IHD as an umbrella term comprises two principal groups of conditions, namely chronic and acute coronary syndromes. It is the latter state that is the main killer globally, whereas those with chronic coronary syndrome have annual mortality of less than $2 \%$ [3]. Yet, the mortality within acute coronary syndrome groups differs drastically as well. Thus, short-term mortality following MI with ST-segment elevation (STEMI) and non-ST-segment elevation MI (NSTEMI) is reported to be significantly higher than that one following UA (7\% vs $3.7-7.4 \%$ vs $0.5-0.7 \%$ ) $[4,5]$, but future non-fatal MI is comparable in UA and NSTEMI withal $(11.2 \%$ vs $7.9 \%$, respectively) [5]. Consequently, UA does need further investigations, especially taking into account the risk of its progression towards MI and the current guidelines recommendations of early discharge after individualized risk stratification [6].

Atherosclerotic plaque disruption and subsequent thrombus formation are considered to be the prevailing processes of atherothrombosis with acute coronary syndrome (ACS) onset. The activation of platelets and the coagulation cascade are the main mechanisms leading to thrombus formation and propagation [7]. As platelets play a crucial role in development of UA and NSTEMI [8] we have focused on platelets. Damage of the endothelium provokes contact of exposed collagen with platelet glycoprotein receptors of different types through von Willebrand factor (vWF) [9]. Platelet activation is attended by the change of its shape and release of adenosine diphosphate, 5-hydroxytryptamine (serotonin), and thromboxane $\mathrm{A}_{2}$. Adenosine triphosphate, ectonucleoside triphosphate diphosphohydrolase-1, C-type lectin-like receptor 2, disintegrin and metalloprotease with a thrombospondin type 1 motif 13 (ADAMTS-13) are other modulators of platelet activation. VWF/ADAMTS-13 axis is one more link that controls the intensity of thrombus formation [7].

Despite the mechanism of plaque instability, thrombus formation and propagation have been investigated thoroughly, the prognostic markers of IHD progression from stable angina (SA) toward UA and further MI remains obscure.

Objective: Our study was aimed to investigate the changes of serotonin and $\mathrm{vWF}$ concentrations among UA patients with and without progression toward MI and to assess the observed parameters as prognostic markers for UA transformation into MI.

\section{Materials and Methods}

\section{Study design}

In this observational cohort study, we enrolled patients who were hospitalized to the cardiology department with UA and out-hospital patients with SA (as the control group).

We followed the guidelines of the European Society of Cardiology 2020 [6] and 2019 [10] to establish the diagnosis and manage the patients with UA and SA, respectively. Thus, we used three groups of criteria: complaints, electrocardiogram (ECG) at rest, biochemical markers (quantitative high-sensitive cardiac Troponin I (hs-cTnI) test). Regarding the latter, we checked it only in UA patients at admission as one of the MI exclusion criterion. SA patients had unchanged ECG (in comparison with the ECG archive of the patient), no complaints or any other signs typical of ACS. Consequently, there were no need in hs-cTnI control. We collected the blood for observed hemostatic parameters analysis before treatment onset (UA patients) or after recruitment (SA patients). All UA patients received standard treatment, namely anticoagulant, acetylsalicylic acid, clopidogrel, high dose of statin, nitrates, $\beta$-blocker (depending on heart rate), and angiotensinconverting enzyme inhibitor (for blood pressure correction), while SA patients were continuing basic treatment. We took blood samples for routine analysis on the next day after admission or recruitment (in case of SA patients) after a 12-hour fasting period. Also, we performed two-dimensional transthoracic echocardiography (2D-TTE). We registered ECG at rest in dynamic at least twice a day and on-demand in UA patients.

While most of the UA patients were treated with good results $(87.7 \%$ ) (Group 2), the clinical condition of $10 \mathrm{UA}$ patients worsened on the 3rd-5th day of treatment $(12.3 \%)$ (Group 3). ECG changes associated with complaints and significantly increased hs-cTnI level (128 (94-301 pg/ml) confirmed the MI onset. STEMI was registered in 1 patient, while NSTEMI was diagnosed in 9 patients. These patients were transferred to a specialized clinic.

\section{Population}

Finally, we enrolled 103 patients with a median age of 65.0 (59.0-69.0) years, 45 females (43.7\%).

After a 28-day follow-up period, we defined three groups: Group 1 - SA patients ( $\mathrm{n}=22$ ), Group 2 - UA patients without progression toward MI $(n=71)$, and Group 3 - UA patients with $\mathrm{MI}$ as a complication $(\mathrm{n}=10)$.

The exclusion criteria were conditions with significant influence on the hemostatic system as well as troponin level such as the history of MI or stroke, heart defects, persistent form of atrial fibrillation/ atrial flutter, cardiomyopathies, nonischemic myocardial injuries, heart failure IIB-III stage, blood diseases including coagulopathies, anemia of II-III stage, severe renal dysfunction (glomerular filtration rate GFR $<30$ $\mathrm{ml} / \mathrm{min} . / 1.73 \mathrm{~m}^{2}$ ), hepatic dysfunction, malignancy, recent (within 6 months) traumas and bleedings, endocrinological disorders, active infection, chronic diseases in the period of exacerbation. 


\section{Blood analysis}

At admission before treatment onset venous whole blood samples were drawn by phlebotomy in sodium citrate $(38 \mathrm{~g} / \mathrm{l}$ at the final ratio of 9:1 vol/vol) with further centrifugation for 40 minutes at $900 \mathrm{~g}$. Plasma samples were aliquoted and frozen at $-80^{\circ} \mathrm{C}$ until use. We analyzed the concentrations of vWF by enzyme-linked immunosorbent assays with primary and secondary antibodies from Santa Crus Biotechnology, CA, USA according to the manufacturer's instructions. We assayed the concentration of serotonin by the ion-exchange chromatography on the KM-Sepharose method [11] with further measurements of serotonin on fluorescence spectrophotometer at an excitation wavelength of $295 \mathrm{~nm}$ and absorption wavelength of $550 \mathrm{~nm}$ against samples containing distilled water [12].

A set of routine analyses was performed by the laboratory of the hospital. Samples were collected after an overnight fast. The laboratory equipment was calibrated.

Hs-cTnI was analyzed by chemiluminescent immunoassay (reference ranges $-<57.27 \mathrm{pg} / \mathrm{ml}$ for males, $<36.99 \mathrm{pg} / \mathrm{ml}-$ for females).

Glomerular filtration rate (GFR) was calculated using the CKD-EPI formula.

Body mass index (BMI) was defined as weight in kilograms divided by height in meters squared.

\section{Instrumental procedures}

We recorded 12-lead surface resting-ECG manually at a sweep of $25 \mathrm{~mm} / \mathrm{s}$. We paid attention to ST-segment depression or elevation, pathological T-wave inversion, new-onset left bundle branch block, arrhythmias. ST-segment elevation was defined as a J-point elevation of $\geq 2 \mathrm{~mm}$ in precordial leads and $\geq 1 \mathrm{~mm}$ in limb leads. ST-segment depression was defined as a J-point decline of $\geq 1.5 \mathrm{~mm}$ in precordial leads and $\geq 1 \mathrm{~mm}$ in limb leads. We referred horizontal, downsloping ST-segment depression to ischemic changes on ECG. Discordant T-waves detected in 2 anatomically contiguous leads were considered a sign of post-ischemic changes.

We measured the blood pressure of patients three times in the admission department using standardized electronic measuring instruments.

2D-TTE was conducted by one expert sonologist using the ultrasound unit of the expert class. We used current 2DTTE scanning guidelines by Mitchell et al. [13]. The apical four-chamber and two-chamber views, parasternal short and long axis, thoracic aorta, upper abdominal aorta, and inferior vena cava were visualized. Also, we assessed wall movements. Valves were evaluated with color Doppler imaging.

\section{Statistical analysis}

We used SPSS (version 22.0, IBM Corp, USA) for data analysis. Continuous variables were reported as medians (interquartile range) (Me (IQR)), and categorical variables as absolute numbers and percentages. For comparison of continuous variables among three groups, we used the KruskalWallis test with further post-hoc Mann-Whitney test with Holm-Bonferroni correction for paired comparison. The $\chi^{2}$ test was used for categorical variables. A binary logistic regression was developed to check if observed parameters as independent variables may be useful for the identification of UA patients with progression toward MI. To create the models, we used the "enter" method. For model fit statistics, we included results of the chi-square likelihood ratio test, pseudoR-square values, assessment of the classification tables, regression weight coefficient, and defined which independent parameter might be useful. Also, we assessed the true positive rate (TPR) and true negative rate (TNR) of each model. Regarding our study, the model's TPR reflected the parameter's prediction accuracy to determine UA patients without risk of progression towards MI. At the same time, the TNR reflected the classifier's ability to detect UA patients with progression toward MI. In other words, TPR and TNR are the sensitivity (Se) and specificity ( $\mathrm{Sp}$ ) of the models respectively. Also, we used bootstrap optimism correction for model validation (simple sampling method, 1000 samples, $95 \%$ confidence interval (CI)).

We constructed receiver operating characteristic curves (ROC-curves) with main points like the area under the curve (AUC) $(95 \% \mathrm{CI}), \mathrm{Sp}, \mathrm{Se}$, and cut-off point.

Finally, we calculated positive likelihood ratios (LR+), which provide medical practitioners with information about the probability of abnormality without depending on the prevalence of abnormality in the study, unlike predictive values [14]. The latter fact was of high-importance in our study as the prevalence of abnormality (UA progression toward MI) was $12.3 \%$.

All tests where possible were 2 -tailed. $\mathrm{P}<0.05$ was considered statistically significant.

\section{Results}

The groups are comparable by baseline characteristics, which are presented in Table 1. The concentrations of observed hemostatic parameters are demonstrated in Fig. 1 and Fig. 2.

In terms of both observed hemostatic parameters, we register no significant difference between Group 1 and Group 2, unlike Group 3 which differs remarkably from both abovementioned cohorts. While serotonin concentration in Group 3 is the highest among the observed groups, vWF concentration is the lowest in this group.

As it is presented in Table 2, both models are significant $(\mathrm{p}<0.001)$ with supposed diagnostic properties. At first sight, serotonin is more accurate in the detection of current abnormality $(80.0 \%$ vs $50.0 \%)$. However, this applies to the detection of patients with UA without MI development, while we are more interested in identifying a group of patients with possible UA progression toward MI. Of note, serotonin and vWF are characterized by opposite directions of the regression coefficient, which is corresponding to Fig. 1 and Fig. 2. 


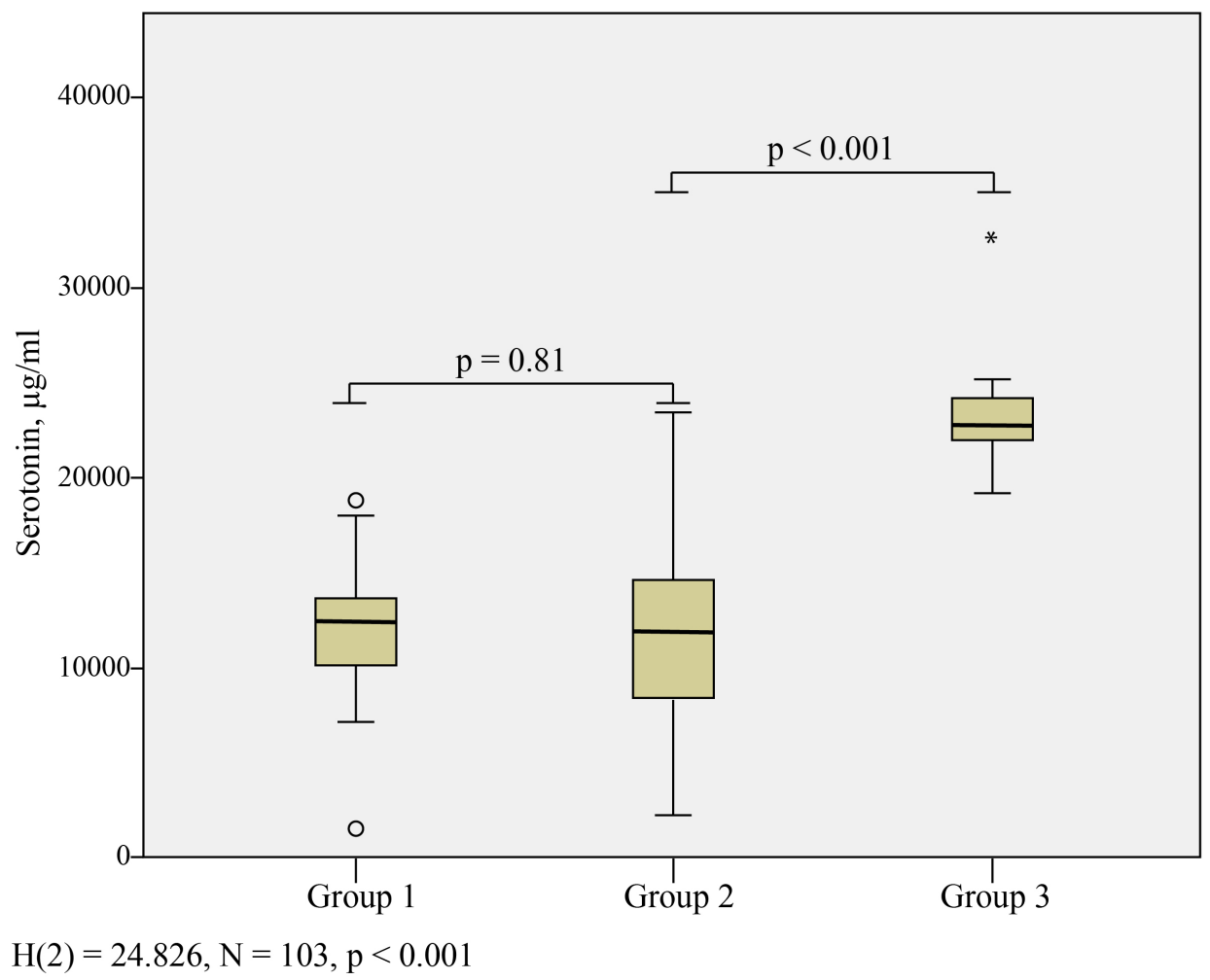

Figure 1. Serotonin concentration among observed groups.

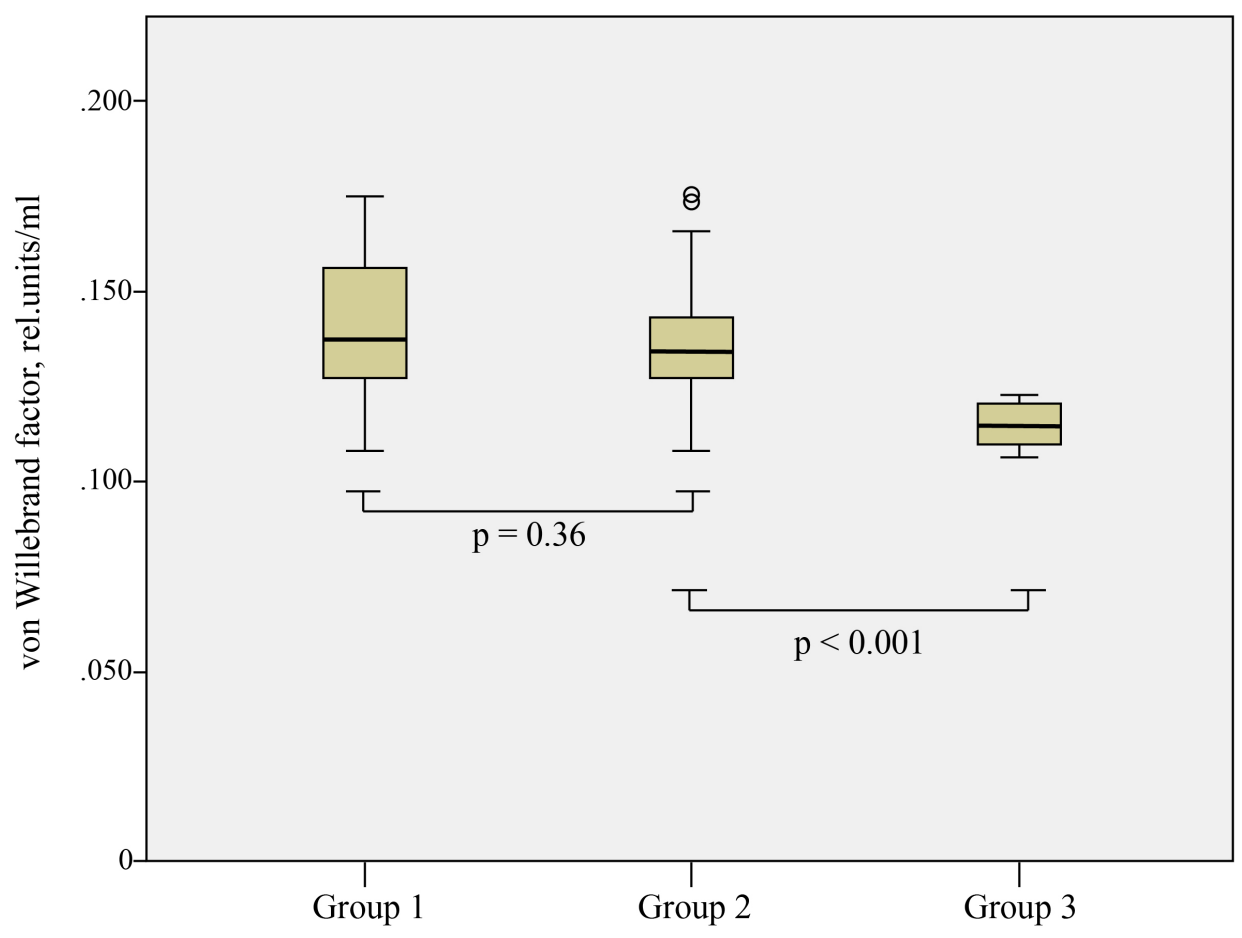

$\mathrm{H}(2)=23.551, \mathrm{~N}=103, \mathrm{p}<0.001$

Figure 2. Factor von Willebrand concentration among observed groups. 
Table 1. Baseline characteristics among the groups.

\begin{tabular}{|c|c|c|c|c|}
\hline Parameter & $\begin{array}{c}\text { Group } 1 \\
n=22\end{array}$ & $\begin{array}{c}\text { Group } 2 \\
n=71\end{array}$ & $\begin{array}{c}\text { Group } 3 \\
n=10\end{array}$ & $\mathrm{p}$ \\
\hline Age, years & $62.5(58.0-69.0)$ & $66.0(60.0-69.0)$ & $61.0(57.0-64.5)$ & 0.19 \\
\hline Females, abs.no (\%) & $11.0(50.0)$ & $31(43.7)$ & $2(25.0)$ & 0.47 \\
\hline BMI, $\mathrm{kg} / \mathrm{m}^{2}$ & $29.4(26.1-32.0)$ & $28.2(25.2-32.2)$ & $29.9(26.1-32.1)$ & 0.42 \\
\hline Smokers, abs.no (\%) & $1(4.5)$ & $13(18.3)$ & $3(37.5)$ & 0.03 \\
\hline Hs-cTnI, pg/ml & - & $25.0(16.0-42.0)$ & $29.0(15.0-48.0)$ & 0.68 \\
\hline $\mathrm{TC}, \mathrm{mmol} / \mathrm{l}^{\#}$ & $5.2(4.5-6.9)$ & $5.1(4.4-6.4)$ & $5.1(4.3-5.5)$ & 0.73 \\
\hline $\mathrm{TG}, \mathrm{mmol} / \mathrm{l}^{\int}$ & $2.6(2.0-3.1)$ & $2.1(1.4-3.0)$ & $2.9(2.2-3.4)$ & 0.08 \\
\hline LDLP, $\mathrm{mmol} / \mathrm{I}^{\#}$ & $2.6(2.2-3.9)$ & $3.1(2.4-3.6)$ & $2.2(1.9-3.0)$ & 0.45 \\
\hline HDLP, mmol// ${ }^{\#}$ & $1.4(1.1-1.8)$ & $1.2(1.0-1.7)$ & $1.0(0.7-1.2)$ & 0.06 \\
\hline ALT, IU/l & $35.0(25.0-55.0)$ & $50.0(45.0-60.0)$ & $45.0(40.0-60.0)$ & 0.29 \\
\hline AST, IU/l & $35.0(30.0-46.0)$ & $35.0(30.0-40.0)$ & $30.0(25.0-45.0)$ & 0.44 \\
\hline $\mathrm{GFR}, \mathrm{ml} / \mathrm{min}$ & $59.0(49.0-70.0)$ & $61.0(49.5-76.0)$ & $60.0(52.6-77.0)$ & 0.57 \\
\hline Glucose, $\mathrm{mmol} / \mathrm{l}^{\mathbb{I}}$ & $4.8(4.0-5.0)$ & $4.4(3.9-5.2)$ & $4.7(3.8-5.4)$ & 0.36 \\
\hline $\mathrm{WBC}, \times 10^{9} / 1$ & $7.6(6.7-8.1)$ & $7.8(6.8-9.3)$ & $8.1(7.1-9.1)$ & 0.06 \\
\hline $\mathrm{RBC}, \times 10^{12} / 1$ & $4.69(4.27-5.15)$ & $4.69(4.31-5.10)$ & $4.48(4.23-5.00)$ & 0.76 \\
\hline $\mathrm{Hb}, \mathrm{g} / \mathrm{l}$ & $142.0(131.0-148.0)$ & $145.0(135.0-165.0)$ & $143.0(134.0-160.0)$ & 0.50 \\
\hline Platelets, $\times 10^{9} / 1$ & $304.0(244.0-336.0)$ & $250.0(207.0-329.0)$ & $280.0(195.0-352.0)$ & 0.34 \\
\hline ESR, mm/hour & $10.0(7.0-15.0)$ & $15.0(7.5-19.0)$ & $16.0(7.9-20.0)$ & 0.16 \\
\hline $\mathrm{ESV}, \mathrm{ml}$ & $45.5(29.0-54.6)$ & $49.5(41.0-62.0)$ & $50.3(42.7-63.0)$ & 0.37 \\
\hline $\mathrm{EDV}, \mathrm{ml}$ & $109.3(95.0-132.0)$ & $118.0(98.8-137.0)$ & $115.0(100.0-133.0)$ & 0.58 \\
\hline $\mathrm{EF}, \%$ & $59.0(57.5-63.0)$ & $57.3(50.7-62.0)$ & $55.5(51.3-61.8)$ & 0.09 \\
\hline IVSd, mm & $1.1(0.9-1.3)$ & $1.2(1.1-1.3)$ & $1.1(1.0-1.3)$ & 0.72 \\
\hline SBP, mmHg & $134.0(125.0-137.0)$ & $145.0(130.0-160.0)$ & $147.0(135.0-167.5)$ & 0.03 \\
\hline $\mathrm{DBP}, \mathrm{mmHg}$ & $79.0(74.0-82.0)$ & $81.0(74.0-92.0)$ & $80.0(76.0-94.0)$ & 0.06 \\
\hline $\mathrm{HR}$, beats/minute & $72.0(67.7-77.5)^{*}$ & $76.0(70.0-85.0)$ & $82.0(78.5-98.0)$ & 0.006 \\
\hline
\end{tabular}

Notes: $\mathrm{p}$ - probability (Kruskal-Wallis H test except hs-cTnI where Mann-Whitney U test was used); ${ }^{*}$ - significant difference between Group 1 and Group 3 (post-hoc Mann-Whitney test with Holm-Bonferroni correction);

BMI - body mass index; Hs-cTnI - high sensitivity cardiac troponin I; TC - total cholesterol; TG - triglycerides; LDLP - low-density lipoprotein cholesterol; HDLP - high-density lipoprotein cholesterol; ALT - alanine aminotransferase; AST - aspartate aminotransferase; GFR - glomerular filtration rate; WBC - white blood cells, RBC - red blood cells, Hb - hemoglobin, ESR - erythrocyte sedimentation rate; ESV - end-systolic volume; EDV - end-diastolic volume; EF - ejection fraction; IVSd - interventricular septum thickness at end-diastole; $\mathrm{SBP}$ - systolic blood pressure; DBP - diastolic blood pressure; HR - heart rate.

Converting indexes: ${ }^{\#}-\mathrm{mg} / \mathrm{dl}=\mathrm{mmol} / \mathrm{l} \times 38.66 ;{ }^{\int}-\mathrm{mg} / \mathrm{dl}=\mathrm{mmol} / \mathrm{l} \times 88.5 ;{ }^{\Upsilon} \mathrm{l}-\mathrm{mg} / \mathrm{dl}=\mathrm{mmol} / \mathrm{l} \times 18.02$.

Afterward, we constructed and ROC-curves (Fig. 3 and Fig. 4) and determined the main characteristics of the latter (Table 3). Models with both serotonin and vWF were shown to be suitable for the detection of patients with a risk of UA transformation toward MI.

Thereafter, we aimed to analyze the utility of vWF and serotonin for early rule-in pathways for UA progression toward MI. For this purpose, we calculated LR+ with posterior probability. These practical aspects are displayed in Table 3.

Thus, serotonin concentration above $21.575 \mu \mathrm{g} / \mathrm{ml}$ increases the probability of UA progression toward MI by $60.7 \%$ (LR+ 19.0), while concerning the decline in vWF concentration less than 0.114 rel.units $/ \mathrm{ml}$ may be a significant predictor of the outcome (LR+18.0).

Besides, taking into account the Se and Sp of indicators as well as LR+, it looks like both factors are better applied to rule-in the patients with UA progression toward MI, unlike to rule out.

\section{Discussion}

Overall, two points are worthy to be highlighted. A significant decrease in vWF concentration and increase in serotonin concentration are typical of UA patients with progression toward MI. Both indicators have demonstrated a high ability to rule in UA patients with risk of transformation into MI.

Enormous studies are dedicated to the issue of MI occurrence in IHD patients, particularly early MI diagnostic [1519]. However, there is limited data regarding risk factors for UA transformation toward MI [20]. Currently, the hs-cTnI test is under precise attention [21]. Implementation of this test has changed the proportion of misdiagnosis among UA and NSTEMI [22]. Chapman et al. asserted that the combination of hs-cTnI with clinical risk scores demonstrated some 
Table 2. Characteristics of binary logistic regression models.

\begin{tabular}{lccccc}
\hline Predictor & Nagelkerke $\mathrm{R}^{2}, \%$ & TPR, \% & TNR, \% & B [95\% CI $]$ & $\mathrm{p}$ \\
\hline Serotonin & 74.0 & 80.0 & 95.8 & $0.708[0.478-3.310]$ & 0.001 \\
vWF & 61.9 & 50.0 & 97.2 & $-248.982[-2046.878-(-180.896)]$ & 0.001 \\
\hline
\end{tabular}

Notes: vWF - von Willebrand factor, $\mathrm{R}^{2}$ - coefficient of determination, TPR - true positive rate,

TNR - true negative rate, B - regression coefficient, CI - confidence interval, $\mathrm{p}$ - probability.

Table 3. The utility of observed predictors as biomarkers of unstable angina progression toward myocardial infarction.

\begin{tabular}{lcccccc}
\hline Indicator & $\mathrm{Se}, \%$ & $\mathrm{Sp}, \%$ & $\mathrm{AUC}$ & Cut-off point, units & $\mathrm{LR}+[95 \% \mathrm{CI}]$ & $\mathrm{PP}$ (odds) [95\%CI] \\
\hline Serotonin & 80.0 & 95.8 & 0.975 & $21.575 \mu \mathrm{g} / \mathrm{ml}$ & $19.0[6.0,60.0]$ & $73 \%(2.7)[46 \%, 89 \%]$ \\
vWF & 50.0 & 97.2 & 0.973 & 0.114 rel.units $/ \mathrm{ml}$ & $18.0[3.9,80.0]$ & $72 \%(2.5)[36 \%, 92 \%]$ \\
\hline
\end{tabular}

Notes: vWF -- von Willebrand factor, Se - - sensitivity, Sp -- specificity, AUC - area under the curve, LR+ - positive likelihood ratio, PP -- posterior probability, CI -- confidence interval.

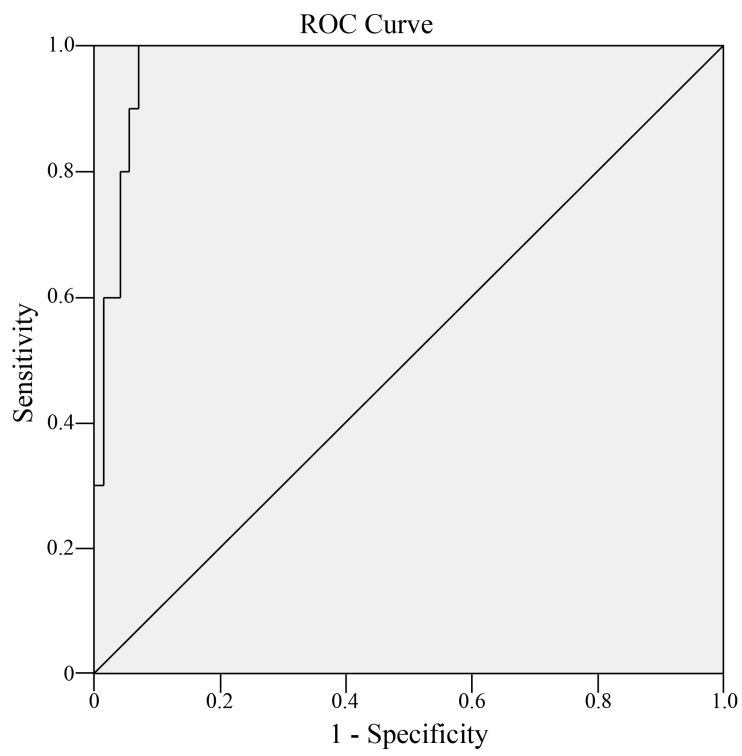

Figure 3. ROC curve with serotonin as an indicator.

benefits to rule in and rule out MI [21], while Giannitsis et al. proclaimed the need for additional risk stratification including other risk indicators [23]. Thus, estimated cardiovascular risk is believed to be more important for accurate clinical management of UA patients [22, 23]. A special feature of our study is the analysis of serotonin and vWF concentration among the patients with already diagnosed UA and further short-term progression toward MI. We revealed the potential usefulness of serotonin and vWF concentrations as prognostic markers in UA patients.

Serotonin [24-33] and vWF [34-40] are still in eyeshot of researchers, albeit of the long-standing history of these substances' investigations [41-45].

The results of studies aimed to investigate blood plasma serotonin concentration are rather ambiguous. Kurano et al.

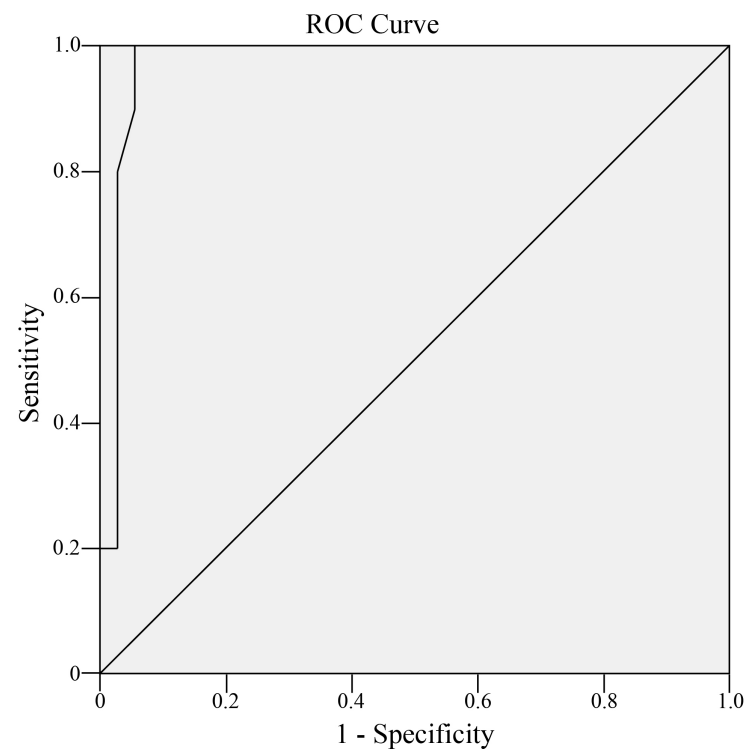

Figure 4. ROC curve with factor von Willebrand as an indicator.

found no significant difference between patients with SA, acute coronary syndrome, and even in a group with no IHD [24]. Rieder et al. revealed no association of serotonin with the severity of coronary artery disease (STEMI) [25]. At the same time, it was shown that serotonin is involved in the serotoninoxidative stress axis which is engaged in the atherothrombosis progression [26].

In our study, the increase in blood plasma serotonin in patients with the outcome may be connected with enhanced activation of platelets which arranges conditions for thrombogenesis. Ziu et al. revealed a link between elevated plasma serotonin, abnormal intracellular serotonin signaling, and accentuated platelet aggregation [41]. Besides, the active involvement of serotonin in a wide range of processes may cause 
such changes. For instance, serotonin is a well-known neurotransmitter with its impact on a variety of psychiatric diseases and behavioral traits [30, 31], including depression [32, 33]. According to Sanner et al. review, depression is an independent risk factor for coronary artery disease throughout platelet serotonin and may be associated with major adverse coronary events (MACE) [42], which may be connected with higher serotonin receptor density or increased platelet response to serotonin [29].

Odaka et al. considered an increase in serotonin above the cut-off point as a marker for coronary microvascular dysfunction [27]. Consequently, the management of UA patients with increased risk of $\mathrm{MI}$ is another disputable issue. It was shown that coronary microvascular dysfunction leads to incomplete perfusion after angiography in about half of the patients [46].

The interaction between serotonin and its specific transporter SERT is another link of the mechanism. Hence, reduced density of SERT molecules, which clear plasma of serotonin, may be connected with a slow decrease in plasma serotonin concentration and leads to thrombotic events [28].

Regarding vWF concentration, we have obtained rather controversial results. Thus, a meta-analysis of 15 studies (960 patients with MACE and 3224 controls without adverse events) revealed that plasma vWF level, namely its elevation, in CAD patients might be an independent prognostic factor for MACE. Remarkably, vWF was significantly higher in patients with MACE unlike without the latter if examined at $24 \mathrm{~h}$ and $48 \mathrm{~h}$ after admission but not on admission. Also, the major heterogeneity in vWF plasma levels at $24 \mathrm{~h}$ after admission was detected due to the utilization of antiplatelet or anticoagulation. In addition, many types of clinical disease which were included contributed to clinical heterogeneity. Furthermore, the timing of MACE occurrence (short-term or long-term) was relatively unclear [34]. Previous studies demonstrated that elevated vWF levels are an independent predictor of MACE in CAD patients over the next 1 year [43]. Anyway, it is worthy to highlight the differences in the design of the mentioned studies including the current one. We suppose that in our study patients with UA transformation into MI were characterized by a longer period of UA duration before treatment onset. Consequently, this fact may cause vWF exhaustion. Vorm et al. registered a decrease in $\mathrm{vWF}$ after repeated bouts of exercise, suggesting either adaptation of endothelial activation or exhaustion of endothelial vWF supplies [47]. Besides, it should be mentioned that vWF is proteolytically degraded by the enzyme ADAMTS13 [35]. Yet, the latter role in cardiovascular disorders is unclear. ADAMTS13 levels were shown to be decreased in MI patients [44], while its increased level was associated with elevated MI risk [45]. Also, wall share rate influence on vWF structure, conformation and concentration is another underinvestigated point $[37,38]$ with some controversial results [37, 39]. Furthermore, the activity of ADAMTS13 depends on vWF conformation. Globular conformation remains resistant to proteolysis, whereas unfolded $v W F$ exposes the binding sites for ADAMTS13 on the A2 domain leading to the cleavage of vWF multimers [37]. Taking into consideration mechanochemistry of $\mathrm{vWF}$ it seems the tight interrelationship between not only vWF an ADAMTS13 but wall share rate exist [40].

A relatively small number of patients, namely in Group 3, is among the limitations of our study, which is partially connected with the prevalence of observed phenomena. However, we followed the exclusion criteria and 47 patients with concomitant disorders were excluded. Also, no angiography was performed in our clinic, but all patients of Group 3 passed angioplasty with stent placement.

\section{Conclusions}

We have detected the high utility of blood plasma serotonin and vWF concentrations for the rule-in the patients with the risk of UA transformation into MI. This fact calls for further researches and may have practical implementation for stratification of the patients with UA.

\section{Prospects of Further Researches}

It is reasonable to study the relations between observed substances serotonin and vWF and linked ones, namely tryptophan and ADAMTS13, respectively, as well as wall share rate to elucidate possible pathogenetic mechanisms. Additionally, the economic utility of biomarkers should be assessed. Hopefully, our results will be used for further researches with further optimization of risk factors stratification in UA patients.

\section{Ethical Statement}

This study is a fragment of the scientific-research work of the Internal Medicine \#4 Department Bogomolets National Medical University "Hemodynamic and coronary blood supply disturbances and ectopic myocardial activity in patients with ischemic heart disease and diabetes mellitus, methods of correction", \#0117U006000 in collaboration with Department of Biochemistry, Educational and Scientific Centre 'Institute of Biology and Medicine", Taras Shevchenko National University of Kyiv, Kyiv, Ukraine (Agreement \#174 of May 27, 2015).

The research was conducted according to WMA Declaration of Helsinki "Ethical principles for medical research involving human subjects".

The study was reviewed and approved by the local institutional Research Ethics Committee of the Bogomolets National Medical University (protocol \#122 of May 29, 2019).

\section{Informed Consent}

The patients gave written consent to participate after the explanation of the survey design. 


\section{Conflict of Interest}

The authors declare that no conflicts exist.

\section{Financial Disclosure}

The authors declared no financial support.

\section{References}

[1] World Health Organization. The top 10 causes of death [Internet]. Available from: https://www.who.int/newsroom/fact-sheets/detail/the-top-10-causes-of-death

[2] Moran AE, Forouzanfar MH, Roth GA, Mensah GA, Ezzati M, Flaxman A, et al. The Global Burden of Ischemic Heart Disease in 1990 and 2010. Circulation [Internet]. 2014 Apr 8;129(14):1493-1501. Available from: https://doi.org/10.1161/CIRCULATIONAHA.113.004046

[3] Dugani SB, Moran AE, Bonow RO, Gaziano TA. Ischemic Heart Disease: Cost-Effective Acute Management and Secondary Prevention. Disease Control Priorities, Third Edition (Volume 5): Cardiovascular, Respiratory, and Related Disorders [Internet]. 2017 Nov 17;135-155. Available from: https://doi.org/10.1596/978-1-4648-0518-9_ch8

[4] Alabas OA, Jernberg T, Pujades-Rodriguez M, Rutherford MJ, West RM, Hall M, et al. Statistics on mortality following acute myocardial infarction in 842897 Europeans. Cardiovascular Research [Internet]. 2019 Jul 26;116(1):149-157. Available from: https://doi.org/10.1093/cvr/cvz197

[5] Puelacher C, Gugala M, Adamson PD, Shah A, Chapman AR, Anand A, et al. Incidence and outcomes of unstable angina compared with nonST-elevation myocardial infarction. Heart [Internet]. 2019 Apr 24;105(18):1423-1431. Available from: https://doi.org/10.1136/heartjnl-2018-314305

[6] Collet J-P, Thiele H, Barbato E, Barthélémy O, Bauersachs J, Bhatt DL, et al. 2020 ESC Guidelines for the management of acute coronary syndromes in patients presenting without persistent ST-segment elevation. European Heart Journal [Internet]. 2020 Aug 29; ehaa575. Available from: https://doi.org/10.1093/eurheartj/ehaa575

[7] Asada Y, Yamashita A, Sato Y, Hatakeyama K. Pathophysiology of atherothrombosis: Mechanisms of thrombus formation on disrupted atherosclerotic plaques. Pathology International [Internet]. 2020 Mar 13;70(6):309-322. Available from: https://doi.org/10.1111/pin.12921
[8] Crea F, Libby P. Acute Coronary Syndromes. Circulation [Internet]. 2017 Sep 19;136(12):1155-1166. Available from: https://doi.org/10.1161/CIRCULATIONAHA.117.029870

[9] Kanaji S, Fahs SA, Shi Q, Haberichter SL, Montgomery RR. Contribution of platelet vs. endothelial VWF to platelet adhesion and hemostasis. Journal of Thrombosis and Haemostasis [Internet]. 2012 Aug;10(8):1646-1652. Available from: https://doi.org/10.1111/j.1538-7836.2012.04797.x

[10] Knuuti J, Wijns W, Saraste A, Capodanno D, Barbato E, Funck-Brentano C, et al. 2019 ESC Guidelines for the diagnosis and management of chronic coronary syndromes. European Heart Journal [Internet]. 2019 Aug 31;41(3):407-477. Available from: https://doi.org/10.1093/eurheartj/ehz425

[11] Maksimenko E, Savchenko V. The level of tryptophan and serotonin in the convulsive readiness conditions of cerebrum. Journal KhNU. 2000;(1):44-47. Available from: https://periodicals.karazin.ua/medicine/article/view/7454

[12] Udenfriend S, Weissbach H, Clark CT. The estimation of 5-hydroxytryptamine (serotonin) in biological tissues. Journal of Biological Chemistry [Internet]. 1955 Jul;215(1):337-344. Available from: https://doi.org/10.1016/S0021-9258(18)66041-7

[13] Mitchell C, Rahko PS, Blauwet LA, Canaday B, Finstuen JA, Foster MC, et al. Guidelines for Performing a Comprehensive Transthoracic Echocardiographic Examination in Adults: Recommendations from the American Society of Echocardiography. Journal of the American Society of Echocardiography [Internet]. 2019 Jan;32(1):1-64. Available from: https://doi.org/10.1016/j.echo.2018.06.004

[14] Deeks JJ, Altman DG. Diagnostic tests 4: likelihood ratios. BMJ [Internet]. 2004 Jul 15;329(7458):168-169. Available from: https://doi.org/10.1136/bmj.329.7458.168

[15] Xu W, Wang L, Zhang R, Sun X, Huang L, Su H, et al. Diagnosis and prognosis of myocardial infarction on a plasmonic chip. Nature Communications [Internet]. 2020 Apr 3;11(1). Available from: https://doi.org/10.1038/s41467020-15487-3

[16] Zeitouni M, Clare RM, Chiswell K, Abdulrahim J, Shah N, Pagidipati NP, et al. Risk Factor Burden and Long-Term Prognosis of Patients With Premature Coronary Artery Disease. Journal of the American Heart Association [Internet]. 2020 Dec 15;9(24). Available from: https://doi.org/10.1161/JAHA.120.017712 
[17] Abdu FA, Mohammed A-Q, Liu L, Xu Y, Che W. Myocardial Infarction with Nonobstructive Coronary Arteries (MINOCA): A Review of the Current Position. Cardiology [Internet]. 2020;145(9):543-552. Available from: https://doi.org/10.1159/000509100

[18] Yao J, Xie Y, Liu Y, Tang Y, Xu J. Prediction Factors of 6-Month Poor Prognosis in Acute Myocardial Infarction Patients. Frontiers in Cardiovascular Medicine [Internet]. 2020 Aug 13;7:130. Available from: https://doi.org/10.3389/fcvm.2020.00130

[19] Stone PH, Maehara A, Coskun AU, Maynard CC, Zaromytidou M, Siasos G, et al. Role of Low Endothelial Shear Stress and Plaque Characteristics in the Prediction of Nonculprit Major Adverse Cardiac Events. JACC: Cardiovascular Imaging [Internet]. 2018 Mar;11(3):462-471. Available from: https://doi.org/10.1016/j.jcmg.2017.01.031

[20] Willerson JT, Yao SK, Ferguson JJ, Anderson HV, Golino P, Buja LM. Unstable angina pectoris and the progression to acute myocardial infarction. Role of platelets and platelet-derived mediators. Tex. Heart I. J. 1991;18(4):243-247. Available from: https://pubmed.ncbi.nlm.nih.gov/15227406/

[21] Chapman AR, Hesse K, Andrews J, Ken Lee K, Anand A, Shah ASV, et al. High-Sensitivity Cardiac Troponin I and Clinical Risk Scores in Patients With Suspected Acute Coronary Syndrome. Circulation [Internet]. 2018 Oct 16;138(16):1654-1665. Available from: https://doi.org/10.1161/CIRCULATIONAHA.118.036426

[22] Eggers KM, Jernberg T, Lindahl B. Unstable Angina in the Era of Cardiac Troponin Assays with Improved Sensitivity-A Clinical Dilemma. The American Journal of Medicine [Internet]. 2017 Dec;130(12):1423-1430.e5. Available from: https://doi.org/10.1016/j.amjmed.2017.05.037

[23] Giannitsis E, Biener M, Hund H, Mueller-Hennessen M, Vafaie M, Gandowitz J, et al. Management and outcomes of patients with unstable angina with undetectable, normal, or intermediate hsTnT levels. Clinical Research in Cardiology [Internet]. 2019 Jul 19;109(4):476-487. Available from: https://doi.org/10.1007/s00392-01901529-4

[24] Kurano M, Dohi T, Nojiri T, Kobayashi T, Hirowatari $\mathrm{Y}$, Inoue $\mathrm{A}$, et al. Blood levels of serotonin are specifically correlated with plasma lysophosphatidylserine among the glycero-lysophospholipids. BBA Clinical [Internet]. 2015 Dec;4:92-98. Available from: https://doi.org/10.1016/j.bbacli.2015.08.003

[25] Rieder M, Laumann R, Witsch T, Schanze N, Heger L, Olivier CB, et al. Evaluation of Serum
Serotonin as a Biomarker for Myocardial Infarction and Ischemia/Reperfusion Injury. Applied Sciences [Internet]. 2020 Sep 13;10(18):6379. Available from: https://doi.org/10.3390/app10186379

[26] Sugiura T, Dohi Y, Yamashita S, Hirowatari Y, Fujii S, Ohte N. Serotonin in peripheral blood reflects oxidative stress and plays a crucial role in atherosclerosis: Novel insights toward holistic anti-atherothrombotic strategy. Atherosclerosis [Internet]. 2016 Mar;246:157-160. Available from: https://doi.org/10.1016/j.atherosclerosis.2016.01.015

[27] Odaka Y, Takahashi J, Tsuburaya R, Nishimiya K, Hao K, Matsumoto Y, et al. Plasma concentration of serotonin is a novel biomarker for coronary microvascular dysfunction in patients with suspected angina and unobstructive coronary arteries. European Heart Journal [Internet]. 2017;38(7):489-496. Available from: https://doi.org/10.1093/eurheartj/ehw448

[28] Fraer M, Kilic F. Serotonin. Hypertension [Internet]. 2015 May;65(5):942-948. Available from: https://doi.org/10.1161/HYPERTENSIONAHA.114.05061

[29] Lancellotti S, Sacco M, Basso M, Cristofaro RD. Mechanochemistry of von Willebrand factor. Biomolecular Concepts [Internet]. 2019 Nov 27;10(1):194-208. Available from: https://doi.org/10.1515/bmc-2019-0022

[30] Rubin R. Exploring the Relationship Between Depression and Dementia. JAMA [Internet]. 2018 Sep 11;320(10):961. Available from: https://doi.org/10.1001/jama.2018.11154

[31] Conio B, Martino M, Magioncalda P, Escelsior A, Inglese M, Amore M, et al. Opposite effects of dopamine and serotonin on resting-state networks: review and implications for psychiatric disorders. Molecular Psychiatry [Internet]. 2019 Apr 5;25(1):82-93. Available from: https://doi.org/10.1038/s41380-019-0406-4

[32] Daut RA, Fonken LK. Circadian regulation of depression: A role for serotonin. Frontiers in Neuroendocrinology [Internet]. 2019 Jul;54:100746. Available from: https://doi.org/10.1016/j.yfrne.2019.04.003

[33] Gałecki P, Talarowska M. Inflammatory theory of depression. Psychiatria Polska [Internet]. 2018 Jun 30;52(3):437-447. Available from: https://doi.org/10.12740/PP/76863

[34] Fan M, Wang X, Peng X, Feng S, Zhao J, Liao L, et al. Prognostic value of plasma von Willebrand factor levels in major adverse cardiovascular events: a systematic review and meta-analysis. BMC Cardiovascular Disorders [Internet]. 2020 Feb 10;20(1). Available from: https://doi.org/10.1186/s12872-020-01375-7 
[35] Green D, Tian L, Greenland P, Liu K, Kibbe M, Tracy R, et al. Association of the von Willebrand Factor-ADAMTS13 Ratio With Incident Cardiovascular Events in Patients With Peripheral Arterial Disease. Clinical and Applied Thrombosis/Hemostasis [Internet]. 2016 Jun 17;23(7):807-813. Available from: https://doi.org/10.1177/1076029616655615

[36] Wang X, Zhao J, Zhang Y, Xue X, Yin J, Liao L, et al. Kinetics of plasma von Willebrand factor in acute myocardial infarction patients: a meta-analysis. Oncotarget [Internet]. 2017 Aug 9;8(52):90371-90379. Available from: https://doi.org/10.18632/oncotarget.20091

[37] Okhota S, Melnikov I, Avtaeva Y, Kozlov S, Gabbasov Z. Shear Stress-Induced Activation of von Willebrand Factor and Cardiovascular Pathology. International Journal of Molecular Sciences [Internet]. 2020 Oct 21;21(20):7804. Available from: https://doi.org/10.3390/ijms21207804

[38] Zhang C, Kelkar A, Neelamegham S. von Willebrand factor self-association is regulated by the sheardependent unfolding of the A2 domain. Blood Advances [Internet]. 2019 Apr 1;3(7):957-968. Available from: https://doi.org/10.1182/bloodadvances.2018030122

[39] Oran I, Cinar C, Bozkaya H, Parildar M, Duman S. Reduced Activity of von Willebrand Factor after Flow-Diverting Stent Implantation for Intracranial Aneurysms: A Link to Acquired von Willebrand Disease? American Journal of Neuroradiology [Internet]. 2020 Jan;41(1):140-146. Available from: https://doi.org/10.3174/ajnr.A6343

[40] Lancellotti S, Sacco M, Basso M, Cristofaro RD. Mechanochemistry of von Willebrand factor. Biomolecular Concepts [Internet]. 2019 Nov 27;10(1):194-208. Available from: https://doi.org/10.1515/bmc-2019-0022

[41] Ziu E, Mercado CP, Li Y, Singh P, Ahmed BA, Freyaldenhoven $\mathrm{S}$, et al. Down-regulation of the serotonin transporter in hyperreactive platelets counteracts the pro-thrombotic effect of serotonin. Journal of Molecular and Cellular Cardiology [Internet]. 2012 May;52(5):1112-1121. Available from: https://doi.org/10.1016/j.yjmcc.2012.02.004
[42] Sanner JE, Frazier L. The Role of Serotonin in Depression and Clotting in the Coronary Artery Disease Population. Journal of Cardiovascular Nursing [Internet]. 2011 Sep;26(5):423-429. Available from: https://doi.org/10.1097/JCN.0b013e3182076a81

[43] Montalescot G, Collet JP, Choussat R, Ankri A, Thomas D. A rise of troponin and/or von Willebrand factor over the first $48 \mathrm{~h}$ is associated with a poorer 1-year outcome in unstable angina patients. International Journal of Cardiology [Internet]. 2000 Feb;72(3):293-294. Available from: https://doi.org/10.1016/S01675273(99)00202-8

[44] Kaikita K, Soejima K, Matsukawa M, Nakagaki T, Ogawa H. Reduced von Willebrand factor-cleaving protease (ADAMTS13) activity in acute myocardial infarction. Journal of Thrombosis and Haemostasis [Internet]. 2006 Nov;4(11):2490-2493. Available from: https://doi.org/10.1111/j.1538-7836.2006.02161.x

[45] Chion CKNK, Doggen CJM, Crawley JTB, Lane DA, Rosendaal FR. ADAMTS13 and von Willebrand factor and the risk of myocardial infarction in men. Blood [Internet]. 2006 Oct 19;109(5):1998-2000. Available from: https://doi.org/10.1182/blood-2006-07-038166

[46] Konijnenberg LSF, Damman P, Duncker DJ, Kloner RA, Nijveldt R, van Geuns R-JM, et al. Pathophysiology and diagnosis of coronary microvascular dysfunction in STelevation myocardial infarction. Cardiovascular Research [Internet]. 2019 Nov 9;116(4):787-805. Available from: https://doi.org/10.1093/cvr/cvz301

[47] Van der Vorm L, Huskens D, Kicken C, Remijn J, Roest M, de Laat B, et al. Effects of Repeated Bouts of Exercise on the Hemostatic System. Seminars in Thrombosis and Hemostasis [Internet]. 2018 Oct 5;44(08):710-722. Available from: https://doi.org/10.1055/s-0038-1673619

Received: 2020-12-04

Revised: 2021-01-03

Accepted: 2021-01-12 